\title{
The Possibility of Replacing Puddled Transplanted Flooded Rice with Direct-seeded rice in Central China (Abstract)
}

\author{
Lixiao Nie \\ College of Plant Science and Technology, Huazhong Agricultural University, Wuhan, China \\ Corresponding author: nielixiao@mail.hzau.edu.cn
}

\begin{abstract}
Replacement of puddled transplanted rice (PTR) by direct-seeded rice (DSR) can potentially reduce consumption of resources and decrease emissions of greenhouse gases while maintaining grain yields in central China. However, direct seeding has not been widely adopted in this region. This review was undertaken to better understand the problems and opportunities for replacing PTR with DSR in central China. The seeding rate, crop growth, grain yield, water productivity, nitrogen use efficiency, greenhouse gas emissions and root development was compared between DSR and PTR. With good water management, grain yield of DSR is similar to or higher than yield of PTR while increasing irrigation water productivity and nitrogen use efficiency, reducing greenhouse gas emissions, and decreasing labor requirement. However, problems that include lodging, weak root development, weed infestations and poor crop establishment under drought, waterlogging, or chilling stresses might limit wide-scale adoption of DSR in central China. Varieties bred and selected for direct seeding, guidelines for improved nutrition, water, and weed management practices, and the development of suitable planting machines and sowing management for DSR are needed. In addition, incorporating DSR into the double season rice or ratoon rice systems could be promising strategies to increase rice production in central China.
\end{abstract}

Keywords: Direct-seeded rice, Puddled Transplanted Flooded Rice, central China. 
